\title{
Organizaciones artísticas en México Modelo e indicadores de capital intelectual
}

Laura Verónica

Herrera Franco

Universidad Veracruzana

lvhf@ prodigy.net.mx

\section{Patricia Ramírez}

Hernández

Universidad Veracruzana maymora13@yahoo.com.mx

Graciela May Mora Universidad Veracruzana patyrh@hotmail.com

\section{Resumen}

Disertar acerca de los activos intangibles o activos intelectuales es necesario para toda organización; este trabajo propone un modelo e indicadores evaluándolos considerando el efecto en los distintos recursos empresariales de las organizaciones artísticas en México. El planteamiento es en el sentido de generar un patrón teórico para la evaluación de capital intelectual o activos intangibles que comprenda indicadores como estabilidad y renovación; bloques referentes a capital humano y relación con el medio ambiente; elementos como sistemas, conocimientos, patentes y competencias. El diseño se fundamenta en modelos como el navegador de Skandia, el monitor de activos intangibles (Sveiby, 1997) y el Intelect. A partir de ellos se genera un esquema que por sus características se considera útil y adaptable para organizaciones donde sus activos son principalmente las habilidades, inteligencia, libre expresión y productos de los individuos que la integran. Esta investigación tiene como objetivo el aspecto cualitativo del modelo, sin embargo, su campo de aplicación para evaluar activos es el área de contabilidad y disciplinas afines a la toma de decisiones y la administración de empresas, trasladando para ello el modelo al ámbito cuantitativo.

Palabras clave: activos intelectuales, activos intangibles, organizaciones artísticas, capital intelectual, evaluación del capital intelectual 


\title{
Artistic organizations in Mexico. Model and indicators of intellectual capital
}

\begin{abstract}
To reason about intangible assets or intellectual assets is essential for any organization; this paper proposes a model and indicators, appraising them in terms of their effect on the different resources of the artistic organizations in Mexico. The approach is in the sense of generating a theoretical pattern for the evaluation of intellectual capital or intangible assets encompassing indicators like: stability and renovation; blocks about human capital and relationship with the environment; elements like systems, knowledge, patents, and competences. The design is based on models like Skandia Navigator, Intangible Assets Monitor (Sveiby, 1997), and Intellect. Since them, we generate a scheme which by its nature is considered as useful and adaptable to organizations whose main assets are skills, intelligence, free expression and products from the individuals that integrate them. This research aims to the qualitative aspect of the model, however, its scope for appraising assets is the accounting and related disciplines to making decisions and business management, which implies to shift the model to the quantitative level.
\end{abstract}

Keywords: intellectual assets, intangible assets, artistic organizations, intellectual capital, intellectual capital evaluation.

\section{Introducción}

En todo momento la búsqueda del conocimiento acerca de una mejor forma de operar en las entidades socioeconómicas ha dado la pauta para elaborar propuestas, teorías y metodologías que favorezcan dichas actividades. En el caso de México, precisamente las instituciones responsables de la regulación de organizaciones y sus operaciones han actualizado y enfocado su atención a homogeneizar sus procesos de registro financiero con el resto de la comunidad internacional. Así, en relación con el concepto de activos intangibles o capital intelectual como rubro integrante de los bienes empresariales se ha visto la necesidad de concretar el reconocimiento de sus efectos organizacionales y su registro contable, auxiliándose de modelos de evaluación y de la formulación de postulados básicos como son las Normas de Información Financiera (NIF), las cuales se refieren al conjunto de pronunciamientos normativos, conceptuales y particulares que regulan la información contenida en los estados financieros y 
sus notas, y que son aceptados de manera amplia y generalizada por sus usuarios (Consejo de Información de Normas de Información Financiera, 2009)¹.

Las organizaciones artísticas, como todo ente constituido por bienes y derechos, requiere un modelo que le facilite el reconocimiento, evaluación, control y presentación de sus activos intangibles. Estas instituciones se vinculan con disciplinas relacionadas con las producciones intelectuales y de artículos de uso, con aspectos de naturaleza inmaterial; por lo que son muy susceptibles de ser estudiadas acerca de sus activos intangibles. Éste es el caso de las indicadas en los cuadros 1, 2 y 3 , las cuales se vinculan con lo que en la actualidad se definen como bellas artes: arquitectura, danza, escultura, música, pintura, literatura, cinematografía, fotografía, historieta; algunos autores añaden a la televisión, teatro y moda (Tatarkiewicz, 2002).

En este trabajo se estudiarán instituciones culturales de México por la facilidad de mostrar la propuesta - y no porque el modelo sea excluyente de otros grupos o asociaciones que coexisten fortaleciendo el quehacer artístico en relación con la materia y técnica utilizados- como academias encargadas de preservar el arte como fenómeno cultural, de reglamentar su estudio y su conservación promocionándolo mediante exposiciones y concursos; organismos dedicados a mostrar colecciones; entidades orientadas a la comercialización; o la organización de ferias y exposiciones. En esta investigación se aborda la conceptualización del capital intelectual en organizaciones artísticas. A continuación se presentan algunos modelos para su valuación, los que por sus características se adecuan a la propuesta objeto de estudio sobre el reconocimiento de tales activos intangibles y la necesidad de valorarlos ordenada e íntegramente.

\section{Activos intangibles en organizaciones artísticas}

Todo bien propiedad de una entidad supone la generación de provechos que finalmente se traducirán en rendimientos o beneficios sociales, así como el mantenimiento y desarrollo organizacional.

\footnotetext{
${ }^{1}$ A partir de 2002 se constituyó el Consejo Mexicano de Normas de Información Financiera (CINIF), organismo reconocido por el Instituto Mexicano de Contadores Públicos, integrado por entidades líderes en sectores público y privado, que — con independencia de criterio e integridad - tiene como objetivo emitir la normatividad contable en México.
} 
La escasez de los recursos también hace necesaria la mejor disposición de los bienes o activos, en este caso los intelectuales, definidos por el boletín C-8 de las NIF como aquellos identificables, sin sustancia física, utilizados para la producción o abastecimiento de bienes, prestación de servicios o para propósitos administrativos, que generarán beneficios económicos futuros controlados por la entidad (CINIF, 2009); durante el desarrollo de este documento también se les denominará como capital intelectual o del conocimiento (Palomo, 2003) ${ }^{2}$. En algunos casos, la medición de estos provechos también ha requerido la asignación de escalas ordinales, nominales, o de intervalo, donde se utilizan símbolos para representar conceptos (Rodríguez, 2003). Para el estudio de los bienes intangibles, y con el fin de medir los efectos económicos o monetarios, se utilizan ratios o razones, variaciones absolutas y relativas, de acuerdo con los indicadores propuestos en el modelo y en atención a los conceptos que integran los bienes de capital: marcas, sistemas, derechos de autor, capital humano, procesos, entre otros.

En México no existe un concepto que identifique a las organizaciones artísticas claramente; se puede decir que, como parte de sus características, son instituciones que involucran autores que tienen facultades para efectuar a partir de su talento obras o producciones artísticas con los que el creador da a conocer su personalidad definiendo con sus métodos el término de estética, mostrando así sus intereses hacia ideales, posturas, cualidades o defectos ${ }^{3}$. En otras palabras, nos referimos a instituciones que fomentan o permiten el desarrollo artístico y sus manifestaciones. En México esa gestión la tiene a su cargo, de manera oficial, el Consejo Nacional para la Cultura y las Artes (Conaculta), que fue creado con el fin de coordinar las políticas, organismos y dependencias de carácter cultural como artístico. Asimismo, tiene labores de promoción, apoyo y patrocinio de los eventos que propicien el arte y la cultura. Ahora bien, la clasificación que se nos presenta a partir de esa entidad es la siguiente:

\footnotetext{
${ }^{2}$ Pero entonces surge la problemática de cómo se puede evaluar un beneficio si anteriormente no había un adecuado sistema de registro y cálculo de los activos señalados; incluso en lo relacionado con la presentación de los intangibles en los informes financieros se dificulta y en algunos aspectos diverge el razonamiento económico con respecto a los postulados contables básicos, por ejemplo, la disminución del valor de los recursos intangibles o su amortización contable cuando en lugar de restar su generación de beneficios se incrementa financieramente $a$ posteriori. Todo ello repercute definitivamente en la presentación razonable y objetiva de los datos contables y en la toma de decisiones.

${ }^{3}$ Definición propia basada en Souriau, 1998.
} 
- Bienes culturales: televisión, radio, computadoras, videojuegos, por ejemplo.

- Medios audiovisuales: radio y televisión.

- Recintos culturales: bienes patrimonio de la humanidad, zonas arqueológicas, casas y centros de cultura, museos, librerías, cines y teatros.

Por lo tanto, se puede citar como parte de esta investigación la evaluación de los activos intelectuales del último rubro indicado, los cuales se detallan en los cuadros 1,2 y 3 , así como de otros más que logren reunir las características de entidades artísticas o culturales.

\section{Cuadro 1 \\ Patrimonio en México}

\begin{tabular}{l|c}
\hline \multicolumn{1}{c|}{ Concepto cultural } & Cantidad en México \\
\hline $\begin{array}{l}\text { Bienes inscritos como patrimonio cultural } \\
\text { de la humanidad }\end{array}$ & 27 \\
\hline
\end{tabular}

Fuente: UNESCO, septiembre de 2007

\section{Cuadro 2}

\section{Recintos culturales en México}

\begin{tabular}{l|c}
\hline \multicolumn{1}{c|}{ Concepto cultural } & Cantidad en México \\
\hline Zonas arqueológicas abiertas al público & 173 \\
\hline Casas y centros de cultura & 1828 \\
\hline Total museos & 1123 \\
\hline Teatros & 573 \\
\hline Librerías & 1375 \\
\hline
\end{tabular}

Fuente: Sistema de Información Cultural, Conaculta, agosto de 2007.

\section{Cuadro 3}

\section{Recinto cultural en México}

\begin{tabular}{c|c}
\hline Concepto cultural & Cantidad en México \\
\hline Cines & 502 \\
\hline
\end{tabular}

Fuente: Imcine, con base en información de Nielsen. 2006 


\section{Modelos de evaluación}

Derivado de investigaciones y casos, los modelos de evaluación de activos intangibles han mostrado diferencias en sus indicadores y variables; de hecho una observación al respecto, señalada por Funes y Hernández (2001), es aquélla donde se sugiere que no es posible que por sus características esos bienes se comporten de forma similar y, por lo tanto, la dificultad de estandarizar y unificar un criterio o método que evalúe dos activos en condiciones idénticas. Sin embargo, la búsqueda por expresar el valor de los derechos empresariales ha dado origen a modelos como el navegador de Skandia, el monitor de activos intangibles, el modelo Intelect y balanced business scorecard, entre otros. En ellos, a pesar de las discrepancias que presentan, se observan similitudes en cuanto a su propósito, clasificación de sus elementos objeto de estudio, algunas de sus variables y categorías.

\section{Propuesta de modelo para entidades artísticas}

A partir de ciertos modelos se elabora una metodología de evaluación para capital del conocimiento en entidades artísticas; con ello se pretende que sea una alternativa de medición de sus resultados, la cual vaya incorporando el valor económico de sus efectos en planes como pronósticos y presupuestos, en herramientas de análisis financiero y en el control a través de políticas o auditorías. Las etapas seguidas para su elaboración fueron las siguientes:

- Revisión de modelos actuales que por sus características se adaptaran a la propuesta específica hacia entidades artísticas.

- Análisis de los elementos que requiere un modelo, es decir, bloques, elementos, indicadores, variables.

- Integración de un modelo o esquema que incluya los datos mencionados acorde con las organizaciones artísticas.

- Planteamiento sobre la manera como los indicadores se pudieran evaluar.

El desarrollo de los puntos anteriores se presenta a continuación:

1. Los modelos como el navegador de Skandia (Edvinsson, 1998), estudiado en Suecia para una entidad de seguros financieros, donde por primera vez surge un departamento y dirección general especializados en capital intelectual (www.uam. es/personal_pdi/economicas/.../modelos1_grupo96.doc), es antecedente de esta 
propuesta debido a que centra su atención en el enfoque humano, característica evidente de las entidades artísticas, y su efecto en otras perspectivas como el cliente y los procesos; con ello se intenta vincular los indicadores de activos intangibles con los resultados financieros, incluso como parte de su capital por el valor acumulado de las inversiones que las empresas han realizado en la formación de sus empleados (Edvinsson, 1998).

Asimismo, el patrón monitor de activos intangibles (Sveiby, 1997) —el cual se investigó en empresas suecas dando origen a la primera teoría de las llamadas organizaciones del conocimiento (www.hacienda.go.cr/.../Modelo\%20contable $\% 20 \mathrm{de} \% 20$ medición $\% 20 \mathrm{del} \% 20$ capital $\% 20 \mathrm{~d}$ ), con lo que fundamentó a la comunidad sueca en prácticas para medir el conocimiento con una de sus corrientes Konrad, origen del monitor de activos intangibles - afirma que las personas son el único agente verdadero de las organizaciones, incluso de su riqueza; asimismo, señala que las competencias de sus miembros son capacidades que tienen para actuar ante distintas situaciones y crear tanto activos materiales como inmateriales, circunstancia que se articula nuevamente con el caso de entidades artísticas. De la misma forma, el patrón monitor de activos intangibles posee indicadores de medida que no todos los modelos tienen y permite que su determinación sea de acuerdo con las características de la empresa en estudio.

Otro prototipo es el modelo Intelect (Euroforum, 1997-1998), el cual ha sido aplicado en España de forma práctica a entidades de diversa índole, se ha utilizado en este documento porque admite la personalización e incorpora previamente al capital humano como generador de otros tipos de capital, indicando que la empresa no lo posee definitivamente, pero que forma parte del valor de la organización y, por lo tanto, debe ser considerado como parte de la misma en especial si es intensiva en conocimiento y depende fundamentalmente de su personal. Dicho modelo pretende ofrecer a los gestores información relevante para la toma de decisiones y facilitar información a terceros sobre el valor de la empresa (www.uam.es/personal_pdi/economicas/.../modelos1_grupo96.doc); además, por sus características de constructo, está integrado por bloques y cada uno de ellos tiene sus propios elementos, de forma similar al esquema propuesto.

En relación con el referente normativo propuesto por el CINIF en México, se atendió el reconocimiento de los activos o elementos por ser evaluados como imagen, marcas, tecnología y sistemas. Existen bienes que al no reunir ciertas 
características de control sobre los beneficios económicos futuros que fluyan del mismo, ni poder restringir el acceso a otros sobre dichos beneficios, no pueden considerarse como intangibles. Sin embargo, cabe puntualizar que el objetivo de este documento es precisamente dar alternativas de evaluación a los activos intelectuales, o como se indica en la propuesta del modelo Intelect: mostrar el conjunto de activos de una sociedad que, pese a no estar reflejados en los estados contables tradicionales, generan o generarán valor para la empresa en el futuro (Euroforum, 1998). Por lo tanto, los modelos anteriores son el fundamento del plan de evaluación por su apego y sesgo a las características expuestas, así como por el tema tratado.

2. Distintas son las secciones que integran un modelo; como se mencionó, de acuerdo con los utilizados como base para esta propuesta, se intenta identificar los activos objeto de agrupación y evaluación en una entidad. Además, se pretende de manera deductiva, y según su naturaleza, continuar determinando bloques donde se concentren y asocien dichos conceptos o elementos que presenten cierto grado de variabilidad o cambio. Posteriormente, se busca establecer cuáles serían los indicadores que permitan evaluar a las variables que presenten los elementos.

3. El esquema propuesto (figura 1) tiene como origen el valor de la organización o entidad, el cual se divide en activos tangibles e intangibles o capital intelectual; de éste último aspecto se desprenderían los siguientes tres bloques:

- Estructura interna. Lo conforman personas que integran dicho esquema; facilita la operatividad en la empresa sistematizando y eficientando los flujos de información.

- Capital humano. Se refiere al conocimiento que tienen las personas en la organización, propiedad de quien lo posee, pudiendo existir un acuerdo en su disposición hacia las entidades.

- Relación con el medio ambiente. Se relaciona con la interacción con el exterior, así como con la imagen que proyecta la entidad, buenas relaciones y calidad. 


\section{Figura 1}

Modelo de evaluación de activos intanguibles

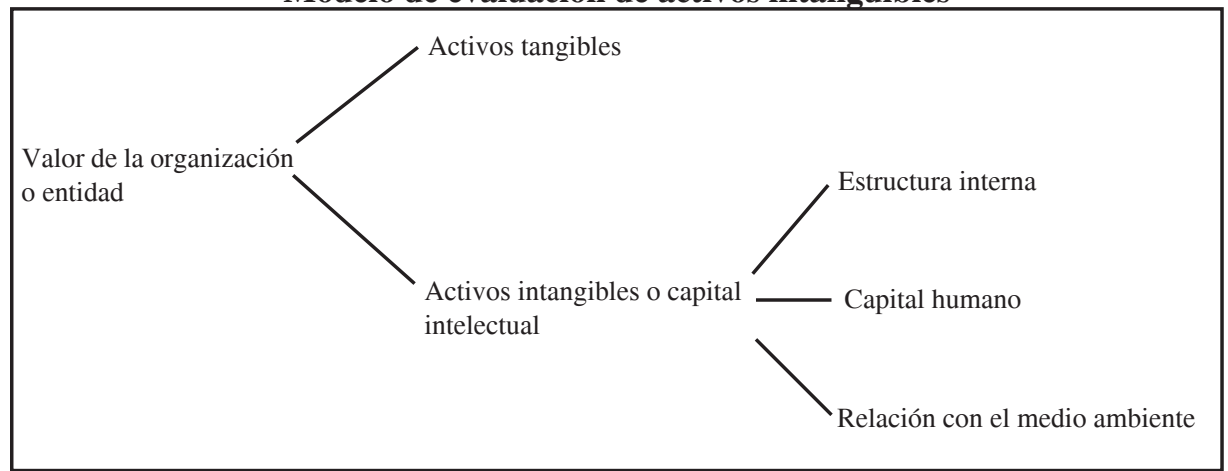

Los activos que son objeto de agrupación se consideran elementos que de acuerdo con su variabilidad afectan a la entidad; su asociación (cuadro 4) es de acuerdo con el modelo propuesto y con la similitud que se encuentra entre los distintos esquemas de valuación existentes.

\section{Cuadro 4}

\section{Bloques y elementos del modelo propuesto}

\begin{tabular}{l|l}
\hline \multicolumn{1}{c|}{ Bloques } & \multicolumn{1}{c}{ Elementos } \\
\hline Estructura interna & $\begin{array}{l}\text { Procesos } \\
\text { Filosofía administrativa } \\
\text { Cultura corporativa } \\
\text { Tecnología } \\
\text { Sistemas }\end{array}$ \\
\hline & $\begin{array}{l}\text { Conocimientos } \\
\text { Habilidades } \\
\text { Competencias } \\
\text { Actitudes } \\
\text { Capital humano }\end{array}$ \\
& $\begin{array}{l}\text { Clientes o mercado } \\
\text { Imagen y marcas } \\
\text { Responsabilidad social }\end{array}$ \\
\hline Relación \\
medio ambiente
\end{tabular}


Se adecuarían los indicadores que pudieran medir o evaluar los bienes intelectuales de las organizaciones artísticas como se muestra en el cuadro 5. A continuación se expone el resumen de esto:

De estabilidad (relacionada con la permanencia). Un activo intelectual se considera estable si se mantiene sin cambios drásticos en el tiempo, o si un cambio de las condiciones iniciales no altera significativamente el futuro del activo intangible. Es posible evaluar la estabilidad a través de la opinión de los trabajadores respecto al ambiente laboral adecuado para que puedan realizar sus funciones - desde las condiciones físicas hasta las condiciones sicológicas del trabajo (http://www. prohumana.cl/)—, así como el otorgamiento de capacitaciones, incentivos, condiciones estructurales acomodadas, rotación de personal, entre otros; también el mantenimiento de estándares adecuados de desempeño y el reconocimiento de los clientes a futuro.

La estructura debe estar en posibilidades de responder, en cierto plazo y bajo ciertos estándares, a los efectos de cambios que se llegaran a dar. En cuanto al posicionamiento en el mercado, éste es posible determinarlo a través del mantenimiento de clientes, antecedentes con proveedores y, en su caso, valor de los títulos de crédito otorgado por la oferta y demanda de su comercialización.

De eficiencia o desempeño (relacionados con la productividad). En relación con la eficiencia, y concibiendo a ésta como la capacidad de disponer de algún recurso para conseguir un efecto determinado, es posible medirla contable y financieramente incluso a través de fórmulas matemáticas, por ejemplo, relacionando utilidades entre inversión para el caso de los beneficios obtenidos por los activos intangibles entre la inversión o el costo de los mismos.

De la misma forma, el desempeño se podría evaluar por medio del porcentaje de competencias que cada persona domina y por el avance en capacitarla en aquéllos que no dominaba. Las competencias son habilidades, educación, experiencia, conocimientos, valores, habilidades sociales. Las entidades del tipo indicado como museos, bibliotecas, casas de cultura, son peculiares en la medición de sus elementos, pues pueden pertenecer a ellas artistas donde su experiencia y capacidad es un bien difícil de controlar o incluso identificar; por ello la propuesta de los indicadores. Por otra parte, la relación con el medio puede medirse a través de la atención que cada organización brinda a la sociedad, y decidir si es adecuada o no. 
Renovación y desarrollo. Si ubicamos estos aspectos como la evolución hacia mejores condiciones, podemos evaluarla en el sentido de si tuvo una adecuada planeación, si se adaptó a su medio ambiente, si tiene la posibilidad de recuperación, modernización y crecimiento; así como la cantidad de proyectos formadores de nuevos artistas, productos, servicios, entre otros.

\section{Cuadro 5}

\section{Propuesta de indicadores}

\begin{tabular}{|c|c|c|c|}
\hline & $\begin{array}{l}\text { Indicador de } \\
\text { estabilidad }\end{array}$ & $\begin{array}{l}\text { Indicador de } \\
\text { eficiencia o } \\
\text { desempeño }\end{array}$ & $\begin{array}{l}\text { Indicador de renovación o } \\
\text { desarrollo }\end{array}$ \\
\hline $\begin{array}{l}\text { Bloque de } \\
\text { estructura } \\
\text { interna }\end{array}$ & $\begin{array}{l}\text { Capacidad de } \\
\text { respuesta } \\
\text { de sistemas y } \\
\text { procesos, tiempos y } \\
\text { costos de solución. }\end{array}$ & $\begin{array}{l}\text { Inversión o costos } \\
\text { en relación con la } \\
\text { recuperación del uso } \\
\text { de sistemas y } \\
\text { tecnología. }\end{array}$ & $\begin{array}{l}\text { Adecuación de la cultura } \\
\text { corporativa al medio, } \\
\text { cumplimiento de planes } \\
\text { estratégicos. } \\
\text { Veces que se presentan } \\
\text { cambios a corto y largo plazo. } \\
\text { Efectos medibles de } \\
\text { reestructuración o cambios. }\end{array}$ \\
\hline $\begin{array}{l}\text { Bloque de } \\
\text { capital } \\
\text { humano }\end{array}$ & $\begin{array}{l}\text { Rotación de personal. } \\
\text { Ambiente laboral y } \\
\text { condiciones de } \\
\text { trabajo. } \\
\text { Mejora en } \\
\text { compensaciones e } \\
\text { incentivos. }\end{array}$ & $\begin{array}{l}\text { Dominio de } \\
\text { competencias y } \\
\text { avance en } \\
\text { capacitación. } \\
\text { Experiencia y } \\
\text { reconocimiento. }\end{array}$ & $\begin{array}{l}\text { Posibilidad de crecimiento } \\
\text { personal y laboral, } \\
\text { cumplimiento de planes } \\
\text { individuales. } \\
\text { Programas originados } \\
\text { dentro de las entidades } \\
\text { artística incubadores de } \\
\text { nuevos artistas, productos } \\
\text { o servicios. }\end{array}$ \\
\hline $\begin{array}{l}\text { Bloque de } \\
\text { relación } \\
\text { con el medio }\end{array}$ & $\begin{array}{l}\text { Mantenimiento de } \\
\text { estándares de calidad } \\
\text { Posicionamiento en el } \\
\text { mercado. }\end{array}$ & $\begin{array}{l}\text { Inversión y } \\
\text { recuperación de } \\
\text { promoción de imagen. } \\
\text { Eficiencia en la } \\
\text { atención de servicios } \\
\text { al público. }\end{array}$ & $\begin{array}{l}\text { Proyectos a los que se } \\
\text { puede tener acceso a través } \\
\text { de convocatorias externas } \\
\text { a la entidad, como es el } \\
\text { caso de los apoyos } \\
\text { gubernamentales. }\end{array}$ \\
\hline
\end{tabular}


A partir de lo anterior, se puede comentar de manera resumida lo referente a la forma de valorar numéricamente los indicadores propuestos (ver cuadro 6) ${ }^{4}$.

Con el fin de complementar el presente trabajo, se expone un estudio de caso elaborado de acuerdo con la propuesta de indicadores de capital del conocimiento y su evaluación, los cuales fueron planteados en los cuadros 5 y 6; sin embargo, el estudio se centra, específicamente, en el bloque de capital humano. Por otra parte, el caso al que se remite es una entidad artística y se estudia su estabilidad, eficiencia o desempeño y renovación o desarrollo. Lo anterior está fundamentado en los elementos distintivos en la definición de un activo intangible según la NIF C-8 y con referencia a las normas internacionales de contabilidad 19, 36 y 38.

Caso de estudio:

Una entidad artística mexicana por decisión de sus directivos y como parte de un plan de negocios realizó un taller de capacitación musical, el cual incluyó a 30 trabajadores que integran los grupos: avanzado uno y dos, finalizando sólo 25 de ellos. Los objetivos esperados, entre otros, fueron los siguientes:

1. Disminuir la rotación de personal al menos en un $5 \%$ anual.

2. Incrementar la cantidad en años de experiencia artística.

3. Cumplir con al menos un $80 \%$ de planes de mejora individual e institucional.

Una vez transcurrido el taller de capacitación y un año posterior al mismo, la propuesta de evaluación del activo intangible Desarrollo por capacitación, según los cuadros 5 y 6 , tuvo los resultados expuestos en el cuadro 7 .

\footnotetext{
${ }^{4}$ Es necesario mencionar que el establecimiento de estándares, razones de proporción, en general indicadores numéricos para efectos de evaluar, sería útil en todos los elementos de la propuesta, los cuales traducidos a políticas institucionales mantendrían no sólo un control, sino posibilitarían la comparación y planeación futura para los tomadores de decisiones en organismos artísticos.
} 


\section{Cuadro 6}

\section{Propuesta de indicadores y su evaluación}

\begin{tabular}{|c|c|c|c|}
\hline & $\begin{array}{l}\text { Indicador de } \\
\text { estabilidad }\end{array}$ & $\begin{array}{l}\text { Indicador de } \\
\text { eficiencia o } \\
\text { desempeño }\end{array}$ & Indicador de renovación o desarrollo \\
\hline $\begin{array}{l}\text { Bloque de } \\
\text { estructura } \\
\text { interna }\end{array}$ & $\begin{array}{l}\text { Ahorro en número } \\
\text { de horas por su } \\
\text { costo en la solución } \\
\text { de problemas. }\end{array}$ & $\begin{array}{l}\text { Inversión monetaria } \\
\text { inicial comparada } \\
\text { con los flujos de } \\
\text { efectivo que } \\
\text { generados en caso } \\
\text { de que sean } \\
\text { identificables con } \\
\text { los sistemas, } \\
\text { tecnología, cultura } \\
\text { corporativa. }\end{array}$ & $\begin{array}{l}\text { Porcentaje de planes artísticos, lúdicos y } \\
\text { organizacionales en general, que se } \\
\text { cumplen. } \\
\text { Número de ciclos, etapas o cambios que } \\
\text { tiene la organización a corto y largo } \\
\text { plazo. } \\
\text { Medición de los efectos de cambio en } \\
\text { unidades monetarias o porcentuales de } \\
\text { la reestructuración o cambios. Posible de } \\
\text { utilizar razones financieras de eficiencia, } \\
\text { rentabilidad sobre activos y valor de } \\
\text { mercado a valor en libros. }\end{array}$ \\
\hline $\begin{array}{l}\text { Bloque de } \\
\text { capital } \\
\text { humano }\end{array}$ & $\begin{array}{l}\text { Razón de rotación de } \\
\text { personal en áreas } \\
\text { localizadas de } \\
\text { entidades artísticas. } \\
\text { Evaluación de } \\
\text { satisfacción laboral } \\
\text { por medio de } \\
\text { cuestionarios. } \\
\text { Posibles de expresar } \\
\text { en escalas ordinales, } \\
\text { medidas estadísticas } \\
\text { e índices. } \\
\text { Variación absoluta o } \\
\text { relativa de cambio en } \\
\text { compensaciones e } \\
\text { incentivos. }\end{array}$ & 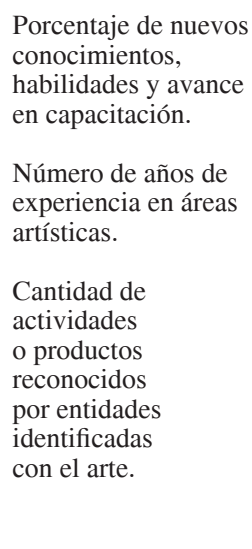 & $\begin{array}{l}\text { Variación absoluta o relativa } \\
\text { de planes individuales } \\
\text { cumplidos en tiempo y } \\
\text { forma. } \\
\text { Cantidad o relación de } \\
\text { programas/plazos generados } \\
\text { internamente en las } \\
\text { organizaciones artísticas } \\
\text { para fortalecer su capital } \\
\text { humano. }\end{array}$ \\
\hline $\begin{array}{l}\text { Bloque de } \\
\text { relación } \\
\text { con el } \\
\text { medio }\end{array}$ & $\begin{array}{l}\text { Tipo y número de } \\
\text { certificación(es) o } \\
\text { recertificación(es) de } \\
\text { calidad, así como su } \\
\text { variación absoluta o } \\
\text { relativa. } \\
\text { Lugar, } \\
\text { mantenimiento, } \\
\text { mejora en la } \\
\text { ubicación dentro del } \\
\text { nicho de mercado } \\
\text { que ocupa la entidad, } \\
\text { a través de escalas } \\
\text { ordinales. }\end{array}$ & $\begin{array}{l}\text { Inversión monetaria } \\
\text { y recuperación a } \\
\text { través de } \\
\text { flujos de efectivo en } \\
\text { promoción de } \\
\text { imagen. } \\
\text { Porcentaje de } \\
\text { eficiencia en la } \\
\text { atención de servicios } \\
\text { al público o en la } \\
\text { venta de productos. } \\
\text { Posible de utilizar } \\
\text { razón financiera } \\
\text { de retorno sobre } \\
\text { servicios prestados. }\end{array}$ & $\begin{array}{l}\text { Cantidad de proyectos a los } \\
\text { que se puede tener acceso } \\
\text { promovidos por organismos } \\
\text { externos. } \\
\text { Variación absoluta o relativa } \\
\text { de nuevos productos o } \\
\text { servicios. }\end{array}$ \\
\hline
\end{tabular}




\section{Cuadro 7}

\section{Resultados de evaluación de activo intangible desarrollo por capacitación}

\begin{tabular}{|c|c|c|c|}
\hline $\begin{array}{l}\text { Relación bloques- } \\
\text { Indicadores }\end{array}$ & $\begin{array}{l}\text { Indicadores de } \\
\text { evaluación }\end{array}$ & $\begin{array}{l}\text { Evaluación actual } \\
\text { (sin activo intangible) }\end{array}$ & $\begin{array}{l}\text { Evaluación del } \\
\text { activo intangible }\end{array}$ \\
\hline $\begin{array}{l}\text { Resultado bloque } \\
\text { capital humano- } \\
\text { indicador } \\
\text { estabilidad }\end{array}$ & $\begin{array}{l}\text { Razón rotación de } \\
\text { personal }\end{array}$ & $\begin{array}{l}\text { Actualmente } 3 \text { bajas } \\
\text { de personal entre } 30 \\
\text { personas al año }=10 \%\end{array}$ & $\begin{array}{l}\text { Una vez que se } \\
\text { registra el desarrollo } \\
\text { por capacitación } 2 \\
\text { bajas de personal } \\
\text { entre } 30 \text { personas al } \\
\text { año }=6.66 \%\end{array}$ \\
\hline $\begin{array}{l}\text { Resultado bloque } \\
\text { capital humano- } \\
\text { indicador } \\
\text { desempeño }\end{array}$ & $\begin{array}{l}\text { Experiencia } \\
\text { artística } \\
\text { en años }\end{array}$ & $\begin{array}{l}\text { Actualmente los } \\
\text { trabajadores varían en } \\
\text { años de experiencia } \\
\text { profesional, sin embargo } \\
\text { todos ellos tienen al } \\
\text { menos cinco. }\end{array}$ & $\begin{array}{l}\text { Un año más de } \\
\text { experiencia } \\
\text { profesional cada } \\
\text { trabajador }\end{array}$ \\
\hline $\begin{array}{l}\text { Resultado bloque } \\
\text { capital humano- } \\
\text { indicador } \\
\text { renovación }\end{array}$ & $\begin{array}{l}\text { Planes cumplidos } \\
\text { individualmente }\end{array}$ & $\begin{array}{l}\text { Los trabajadores } \\
\text { venían cumpliendo y } \\
\text { finalizando sus planes } \\
21 \text { del total de } 30 \\
=70.00 \%\end{array}$ & $\begin{array}{l}\text { Se cumplieron } 25 \\
\text { planes de capacitación } \\
\text { individual del total } \\
\text { de trabajadores que } \\
\text { iniciaron el taller, } \\
\text { un } 83.33 \%\end{array}$ \\
\hline $\begin{array}{l}\text { Resultado bloque } \\
\text { capital humano- } \\
\text { indicador } \\
\text { renovación }\end{array}$ & $\begin{array}{l}\text { Planes cumplidos } \\
\text { institucionalmente }\end{array}$ & $\begin{array}{l}\text { Se planean tres planes al } \\
\text { año y se han realizado } \\
\text { los mismos }=100 \%\end{array}$ & $\begin{array}{l}\text { Se tenían planeados } \\
\text { cuatro planes en un } \\
\text { año, el taller fue uno de } \\
\text { ellos, representando } \\
\text { un } 25 \% \text {. Se } \\
\text { cumplieron todos } \\
\text { ellos, un } 100 \%\end{array}$ \\
\hline
\end{tabular}

Los resultados de acuerdo con los objetivos planeados fueron posibles de evaluar según los indicadores propuestos (cuadro 6) aplicados al taller de capacitación, independientemente si fueron satisfactorios o no para la entidad artística. La propuesta del modelo, entre otros aspectos, favorecería que la organización considerara dichas valuaciones para que el desarrollo por capacitación fuera sujeto de incorporación como activo intangible. Esto se podría indicar como una nota a los estados financieros o en todo caso, aunque no se presentará el activo en el cuerpo de informe, generando una nota como propuesta de evaluación. En cuanto al monto contable sería objeto de otro documento de investigación, éste sólo aplica a indicadores. Lo anterior no pretende contradecir la normatividad contable mexicana; por 
el contrario, busca encontrar las formas de medición, las maneras de presentación financiera para su registro, análisis, planeación y control.

\section{Conclusiones}

El quehacer contable no se limita al único cumplimiento de las disposiciones emitidas por los organismos responsables, también debe procurar la mejor forma de reconocer los bienes concernientes a las entidades económicas. De ello depende que la presentación de los estados financieros sea más objetiva, veraz, confiable y las decisiones se vean enriquecidas con nueva metodología. Es cierto que por sus características es difícil la evaluación de los bienes de capital intelectual y sus beneficios, pero hasta el momento han sido varios los autores e instituciones que han mostrado modelos para tal fin.

Las organizaciones artísticas requieren que sus bienes y los beneficios que otorgan sean tasados de acuerdo con la normatividad vigente y con los modelos de evaluación; en esta propuesta se sugieren tres indicadores con bloques y elementos que organicen dichas ideas, auxiliándose de la teoría existente, pero con modificaciones o adecuaciones a estas agrupaciones en México.

Sería menester de otras investigaciones presentar el modelo anterior aplicado a casos prácticos contables, donde se determinen conceptos y cantidades que permitan la valuación y presentación de los bienes intelectuales en los informes financieros y sus notas.

El modelo, y sus indicadores incluso, puede ser válido para entidades que no son de naturaleza artística. A partir de lo indicado en la propuesta, se visualiza la forma de obtener elementos que se vinculen al reconocimiento monetario y revelación de los activos intelectuales, así como de la posibilidad de compararlos o controlarlos.

Finalmente, es una manera de presentar las necesidades de reconocimiento de capital intelectual y sus respuestas sugeridas, ya que, aunque incorpóreo, es efectivamente un activo generador de bienes que afectan al sector artístico y que, como todos, lo demanda por su misión e interrelación mundial. 


\section{Referencias}

Consejo Mexicano para la Investigación y Desarrollo de Normas de Información Financiera, A.C. (2009). Normas de Información Financiera. México: Instituto Mexicano de Contadores Públicos.

Edvinsson, L. y M. Malone (1998). El capital intelectual: cómo identificar y calcular el valor inexplorado de los recursos intangibles de su empresa. Tr. Jorge Cárdenas Nanetti. Bogotá: Norma.

Funes Cataño, Y. y C. Hernández (2001). Medición del valor del capital intelectual. Contaduría y Administración 203: 45-57.

I.U. Euroforum (1998). Conferencia de la Dirección General de Trabajo y Empleo y Consejería de Economía y Empleo de Madrid. España.

Palomo, M. (2003). La evaluación de activos intangibles. Ingeniería industrial, FCQ-UANL. México. VI (20): 12-17.

Rodríguez, O. (2003). Indicadores de capital intelectual: concepto y elaboración. Madrid: Instituto Universitario de Administración de Empresas, Universidad Autónoma de Madrid.

Sveiby, Karl-Erik (1997). The intangible assets monitor. Journal of Human Resource Costing 2 (1): 73-97.

Souriau, Étienne (1998). Diccionario akal de estética. Madrid: Akal.

Tatarkiewicz (2002). Historia de seis ideas. Madrid: Tecnos.

htt://www.conaculta.gob.mx/

http://www.imcine.gob.mx/. Con base en información de Nielsen, 2006.

http://www.prohumana.cl/

htt://www.unesco.org/ 
http://www.uam.es/personal_pdi/economicas/.../modelos1_grupo96.doc http://www.hacienda.go.cr/.../Modelo\%20contable\%20de\%20medición\%20del\% 20capital\%20d ( 
